\title{
Quantitation of Vessel Morphology from 3D MRA
}

\author{
A. F. Frangi, W. J. Niessen, R. M. Hoogeveen, Th. van Walsum, and \\ M. A. Viergever* \\ Image Sciences Institute, Utrecht Medical Center (UMC) \\ Room E.01.334, Heidelberglaan 100, 3584 CX Utrecht, The Netherlands
}

\begin{abstract}
Three dimensional magnetic resonance angiographic images (3D MRA) are routinely inspected using maximum intensity projections (MIP). However, accuracy of stenosis estimates based on projections is limited. Therefore, a method for quantitative 3D MRA is introduced. Linear vessel segments are modeled with a central vessel axis curve coupled to a vessel wall surface. First, the central vessel axis is determined. Subsequently, the vessel wall is segmented using knowledge of the acquisition process. The user interaction to initialize the model is performed in a $3 \mathrm{D}$ setting. The method is validated on a carotid bifurcation phantom and also illustrated on patient data.
\end{abstract}

\section{Introduction}

Accurate determination of vessel width is necessary for grading vascular stenoses, which is important in diagnosis and treatment planning in, for instance, stroke patients. Studies have revealed that patients with a severe $(>70 \%)$ symptomatic stenosis in the carotids should be operated while patients with stenoses smaller than $30 \%$ should not undergo surgical treatment [1,2].

MRA is increasingly used for vascular diagnosis. Although evaluation by radiologists is mainly performed on 2D maximum intensity projections (MIP) [3], it is known that these lead to vessel width underestimation and decreased contrastto-noise ratio (CNR) $[3,4,5]$.

In order to improve grading of stenoses we suggest a novel scheme for quantitative vessel analysis from 3D MRA. Hereto we use a 3D B-spline deformable model of a vessel segment which consists of a central vessel axis coupled to a vessel wall surface. The use of a B-spline representation for the curve and surface models enables the use of already existing, powerful interaction mechanisms inherited from Computer Assisted Design (CAD). For initialization, which is an important step in segmentation schemes based on deformable models, we suggest a technique based on an iso-surface rendering of the vasculature which allows for intuitive and efficient interaction in a 3D setting. In the fitting procedure,

\footnotetext{
* This research was sponsored by the Dutch Ministry of Economic Affairs (Program IOP Beeldverwerking, Project IBV97009) and EasyVision Advanced Development at Philips Medical Systems Nederland B.V.
} 
information of the image acquisition process is used to accurately define the vessel boundaries. The method is validated on MR phantom data and compared to operator performance. Results on patient data of the carotids are also shown.

\section{Model-Based Vessel Segmentation}

A two-step vessel segmentation procedure is proposed. First, a representation of the central vessel axis is obtained, which is subsequently used as a reference for extracting the boundaries of the vessel.

The central vessel axis, $\mathbf{C}(t)$, is modeled using a B-spline curve of degree $n$ with $s+1$ control points. This representation enforces the lumen line to be connected

$$
\mathbf{C}(t)=\sum_{i=0}^{s} N_{i n}(t) \mathbf{P}_{i}
$$

Here $\mathbf{P}_{i}$ are the control points, $N_{i n}(t)$ is the i-th B-spline basis function of order $n[6]$ and $t \in[0,1]$. The model (sometimes referred to as snake) deforms towards the center of the vessel by minimizing an energy functional, $\mathcal{E}^{C}$, containing terms associated with the smoothness of the spline and the image content $[7,8]$

$$
\mathcal{E}^{C}=\mathcal{E}_{\text {ext }}^{C}+\gamma^{C} \mathcal{E}_{\text {int }}^{C}
$$

where $\mathcal{E}_{\text {int }}^{C}$ provides a regularization term depending on the first and second order parametric derivatives of the curve [9] and is weighted by a factor $\gamma^{C}$. The external energy, $\mathcal{E}_{e x t}^{C}$, is used to attract the curve towards points which have a high likelihood of lying at the central vessel axis. For this purpose, a new filter was previously developed [10] which is based on the eigenvalues $\left(\left|\lambda_{1}\right| \leq\left|\lambda_{2}\right| \leq\right.$ $\left.\left|\lambda_{3}\right|\right)$ of the Hessian matrix computed at each voxel of the image.

$\mathcal{V}(\mathbf{x}, \sigma) \triangleq \begin{cases}0 & \text { if } \lambda_{2}>0 \text { or } \lambda_{3}>0 \\ {\left[1-\exp \left(-\frac{\mathcal{R}_{\mathcal{A}}{ }^{2}}{2 \alpha^{2}}\right)\right] \exp \left(-\frac{\mathcal{R}_{\mathcal{B}}{ }^{2}}{2 \beta^{2}}\right)\left[1-\exp \left(-\frac{\mathcal{S}^{2}}{2 c^{2}}\right)\right]} & \text { otherwise }\end{cases}$

$$
\mathcal{R}_{\mathcal{A}} \triangleq \frac{\left|\lambda_{2}\right|}{\left|\lambda_{3}\right|} \quad \mathcal{R}_{\mathcal{B}} \triangleq \frac{\left|\lambda_{1}\right|}{\sqrt{\left|\lambda_{2} \lambda_{3}\right|}} \quad \mathcal{S} \triangleq \sqrt{\sum_{j} \lambda_{j}^{2}}
$$

The filter can be interpreted as follows. For a bright, tubular structure we expect two eigenvalues which are large, negative, and of similar magnitude, and one eigenvalue which is small. Thus $\mathcal{R}_{\mathcal{B}}$, which is the smallest eigenvalue over the geometric average of the two larger eigenvalues, should be small. Moreover $\mathcal{R}_{\mathcal{A}}$, which measures the anisotropy in the cross-sectional plane should be close to one. Finally, in order to only measure tubular structures above the noise level, $\mathcal{S}$ measures the degree of "image content". The parameters $\alpha, \beta$ and $c$ tune the 
sensitivity of the filter to deviations in $\mathcal{R}_{\mathcal{A}}, \mathcal{R}_{\mathcal{B}}$ and $\mathcal{S}$, relative to the ideal behavior for a line structure ${ }^{1}$.

The Hessian matrix is computed using Gaussian derivative operators at multiple scales, in order to span the range of expected vessel widths of the imaged anatomy $\left(\sigma \in\left[\sigma_{\min }, \sigma_{\max }\right]\right)$. In order to provide a unique filter response, the outputs of the filters at different scales undergo a scale selection procedure [1] -Equation (5). Different vessel sizes can be detected at their corresponding scales; therefore, both small and large vessels will be captured with the same scheme.

$$
\mathcal{V}(\mathbf{x})=\max _{\sigma_{\min } \leq \sigma \leq \sigma_{\max }} \mathcal{V}(\mathbf{x}, \sigma)
$$

The designed filter has the properties that $i$. it filters out non tubular structures, $i$. it is maximum at the center of the vessel while the output of the filter decreases slowly towards the boundaries, and iii. it is sensitive to vessels of different sizes [10]. The filter is therefore suited as an image force to attract the curve towards the central vessel axis. The external energy we propose is given by

$$
\mathcal{E}_{\text {ext }}^{C}=-\frac{1}{\ell} \int_{0}^{1} \mathcal{V}(\mathbf{C}(t))\left\|\partial_{t} \mathbf{C}(t)\right\| d t
$$

where $\ell$ denotes the length of the central vessel axis.

Once the central vessel axis is computed, we proceed to determine the vessel wall. Hereto, the vessel wall is modeled using a tensor product B-spline surface $[12]$

$$
\mathbf{W}(t, u)=\sum_{j=0}^{q} \sum_{k=0}^{r} N_{j l}(u) N_{k m}(t) \mathbf{P}_{j k}
$$

where $\mathbf{P}_{j k}$ are $((q+1) \times(r+1))$ control points, $N_{j l}(u)$ is the $\mathrm{j}$-th B-spline periodic basis function of order $l$, and $u \in[0,2 \pi) ; N_{k m}(t)$ is the k-th B-spline nonperiodic basis function of order $m$, and $t \in[0,1]$. The parameters $u$ and $t$ traverse the surface in the circumferential and longitudinal directions respectively. We have deliberately coupled the longitudinal parameter $(t)$ of the vessel wall and the central vessel axis since both span the vessel in the longitudinal direction. This coupling makes it possible to relate central vessel axis points with the corresponding boundary points.

To fit the vessel wall model in a smooth fashion we use an approach similar to the one applied for the central vessel axis, extending the concept from curves to surfaces. The wall model is deformed in a way that minimizes the following energy criterion

$$
\mathcal{E}^{W}=\mathcal{E}_{\text {ext }}^{W}+\gamma^{W} \mathcal{E}_{\text {int }}^{W}
$$

where the internal energy term, $\mathcal{E}_{\text {int }}^{W}$, takes into account the stretching and bending energies of the vessel wall model. The stretching energy term can be physically interpreted as an approximation to the energy of a thin plate under tension

\footnotetext{
${ }^{1}$ For further details of the filter and typical parameter settings we refer to [10].
} 


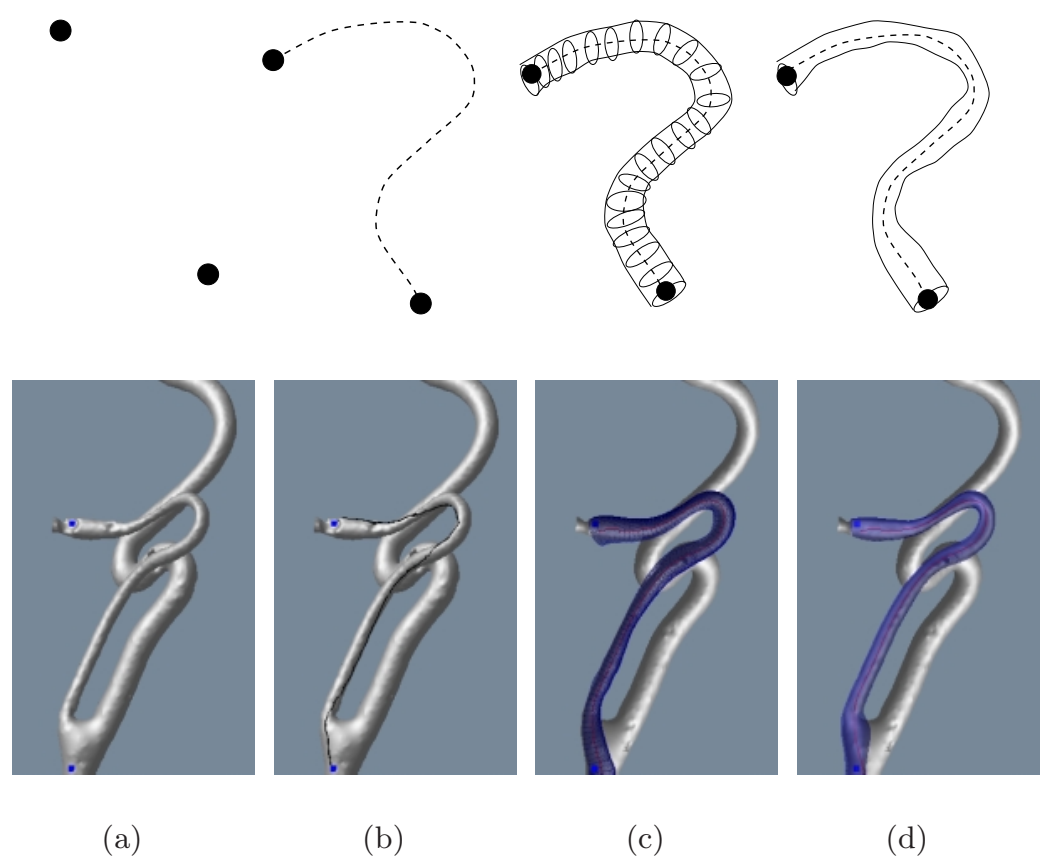

Fig. 1. Interaction scenario. a) A user initializes two (or more) points on the surface. b) From these seeds, a geodesic path is computed. c) The geodesic path is deformed until the central vessel axis is determined. Using the distance between the newly obtained vessel axis and the original geodesic path, a circular cross-section is swept along the axis to generate an initialization of the vessel wall model. d) Vessel wall (after deformation) and central vessel axis.

while the bending energy is related to the rigidity of the deformable surface [9]. Both energies can be expressed as a combination of first and second order parametric derivatives of the surface, respectively.

For the external image force we use knowledge of the MR acquisition protocol. In previous work we showed that the optimal criterion for vessel width estimation depends on the MRA acquisition [13]. If $i$. the resolution is sufficiently high (at least 3 pixels/dim.), ii. saturation due to slow inflow at the borders is limited (for TOF), and iii. flow artifacts are suppressed, the boundaries in Time-OfFlight (TOF) and Contrast Enhanced (CE) acquisitions can be determined using the full-width-half-maximum (FWHM) criterion, while for Phase Contrast (PC) acquisitions the full-width-10\%-maximum (FWTM) should be used (all with respect to the maximum luminal signal) [13]. These criteria can be casted into an energy function in the following way

$$
\mathcal{E}_{e x t}^{W}=\frac{1}{S} \int_{0}^{1} \int_{0}^{2 \pi}\left|\tau_{a c q}-\frac{L(\mathbf{W}(t, u))}{L(\mathbf{C}(t))}\right|\left\|\partial_{t} \mathbf{W} \times \partial_{u} \mathbf{W}\right\| d t d u
$$


where $L(\mathbf{x})$ is the image grey-level at position $\mathbf{x}, S$ is the vessel wall area, and $\tau_{a c q}$ is a threshold that introduces the knowledge about the type of MRA imaging technique. This constant equals 0.5 for TOF and CE, and 0.1 for PC MR angiography. Notice that the weight $\left\|\partial_{t} \mathbf{W} \times \partial_{u} \mathbf{W}\right\|$ takes into account possible departures from chord-length parameterization in the surface.

To start the fitting procedure, first an initialization of the central vessel axis is required. Initialization and interaction of $3 \mathrm{D}$ deformable models is still an open problem. However, proper initialization is a requirement to find the correct local minimum for most deformable model approaches. We suggest the use of an iso-surface rendering for interactively inspecting the vascular anatomy and for selecting the target segment. In Figure 1, the steps of the procedure are highlighted. First, an iso-surface rendering of the angiogram is generated (Figure 1(a)) using marching cubes [14]. Secondly, the operator defines a vessel segment by clicking two points on the iso-surface which define the end-points of a geodesic path (minimum length path on the surface; this is the only step in the algorithm where user interaction is required). This path is used for initializing the central vessel axis after it has been converted into B-spline form using a least squares approximation -Figure 1(b). Thirdly, once the central vessel axis has been determined, the vessel wall is initialized with no extra user interaction by computing a radius function as the distance between the central vessel axis and the geodesic path. This distance provides a rough approximation of the vessel radius at every point along the center line. Using a standard CAD technique known as swept surfaces [12] the vessel model is initialized by sweeping a circle of the estimated radius orthogonally along the central vessel axis-Figure 1(c). Figure 1(d) shows the final result of the fitting procedure.

\section{Results}

In order to assess the performance of the algorithm we addressed the problem of diameter measurements for stenosis grading in an MR compatible, carotid bifurcation phantom with an asymmetric stenosis (R.G. Shelley Lt., North York, Ontario). A photograph of this phantom is shown in the left frame of Figure 2. The phantom is embedded in a rigid, transparent acrylic and manufactured to reproduce normal dimensions in the human vasculature [15].
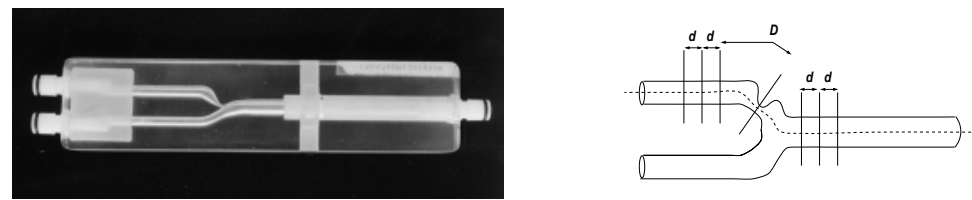

Fig. 2. Left: Carotid bifurcation phantom with an asymmetric stenosis (Courtesy of R.G. Shelley Lt., North York, Ontario, Canada). Right: Manual measurement protocol. In this study we used $D=15 \mathrm{~mm}$ and $d=5 \mathrm{~mm}$. 
Images were acquired on a 1.5 Tesla MR imaging system (Philips Gyroscan ACS-NT, Philips Medical Systems, Best, The Netherlands) with a quadrature head-neck receiver coil. Imaging parameters were as follows. Three-dimensional TOF acquisition: echo time (TE) $1.9 \mathrm{~ms}$, repetition time (TR) $25.0 \mathrm{~ms}$ and flip angle $(\alpha) 15^{\circ}$, slice thickness $1.0 \mathrm{~mm}$. CE acquisition: TE $2.0 \mathrm{~ms}$, TR $6.6 \mathrm{~ms}$, $\alpha 40^{\circ}$, slice thickness $1.0 \mathrm{~mm}$. Both acquisitions: FOV $256 \mathrm{~mm}$, scan matrix $256 \times 256$. Flow was regulated by a computer-controlled pump (Quest Image Inc., London, Ontario). Water was guided through the phantom with constant velocity $(5 \mathrm{ml} / \mathrm{s})$. For CE acquisitions a $5 \mathrm{mM}$ solution of gadopentetate dimeglumine (Magnevist, Schering, Berlin, Germany) was used under the same flow conditions as the $3 \mathrm{D}$ TOF acquisition.

We assessed the performance of the algorithm in comparison with human operators and the ground truth as gold standard. The degree of stenosis was graded by two experts following a manual procedure. This is based on visual inspection of vessel dimensions on a multi planar reformatted (MPR) image generated by manually drawing a central vessel axis and computing the plane perpendicular to it. This procedure was performed on a clinical workstation (EasyVision, Philips Medical Systems, Best, The Netherlands).

In order to compare the measurements provided by the experts and the results obtained with our algorithm, a measurement protocol was defined as indicated in the right frame of Figure 2. The degree of stenosis was computed using the NASCET index (stenosis=1-(minimal residual lumen/distal ICA lumen diameter)x100\%)[1]), and the CC index (with reference taken at the common carotid artery). To incorporate operator variability, the protocol required that the distal diameter was measured at $D=15 \mathrm{~mm}$ from the center of the stenosis and for two other successive planes separated by $d=5 \mathrm{~mm}$. For each plane, the minimum and maximum observed diameters were recorded. All measurements were done twice by the same expert with enough delay to disregard any possible bias in the second measurement. The average stenosis grade and the $99 \%$ confidence interval (CI) were computed for each observer, for both observers and for the proposed algorithm. The statistics of stenosis grading with the model based approach were computed based on all possible values of the degree of stenosis for a region of $2 \mathrm{~mm}$ around the stenosis and a region of $2 d=10 \mathrm{~mm}$ centered at a distance $D+d=20 \mathrm{~mm}$ from the stenosis. This yields a measure of stability of the model-based measurements in the region where the operators performed the manual analysis.

In Figure 3, the average diameter measurements are shown (the average diameter in the orthogonal plane, at a given location along the central vessel axis). For comparison purposes, it also includes, at three points, diameter values from the specifications of the phantom [15].

In Table 3, the statistics of the stenosis grading are summarized. The phantom has a stenosis index of $69.2 \%$ according to NASCET and $78.5 \%$ according to CC (estimated from the manufacturer's specifications [15]). The table shows that the method significantly improves stenosis grading with an absolute error 


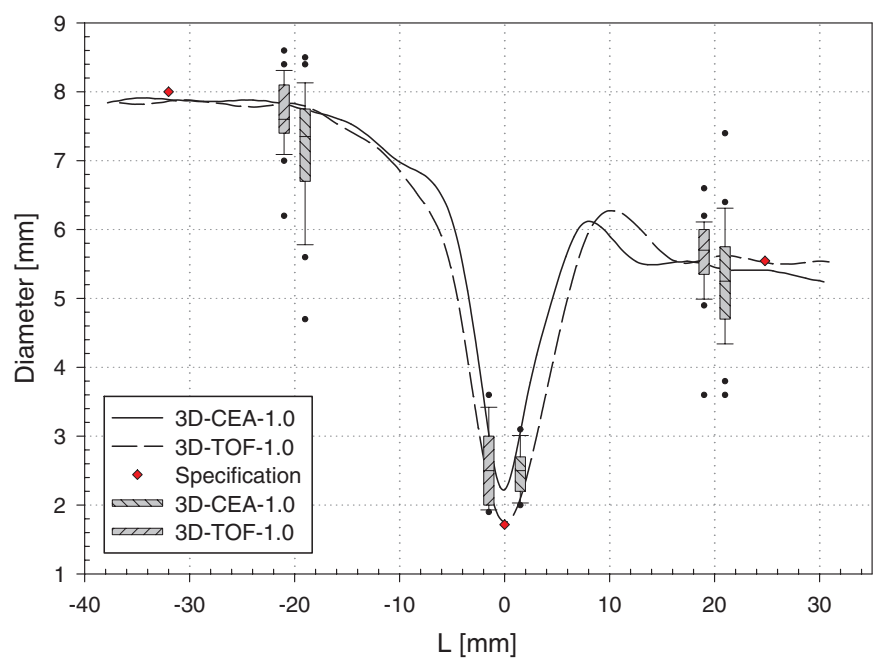

Fig. 3. Average diameter plots for the carotid bifurcation phantom starting at the common carotid artery and upstream to the stenoted branch. The diameter of the phantom (estimated from phantom specifications [15]) and the box-andwhisker plots corresponding to the measurements performed by two experts are also included. Two acquisitions were considered in this experiment, 3D TOF and CE MRA.

smaller than $3.3 \%$ for both criteria. Moreover reproducibility is better in the model based approach.

We also tested the method on patient data. Figure 4(a) and 4(b) show MIPs of $3 \mathrm{D}$ TOF angiograms of the left (mild stenosis) and right (severe stenosis) carotids of the same patient, respectively. Figure 4(c) shows the left carotids of a second patient with a mild stenosis. The in-plane resolution of the $3 \mathrm{D}$ TOF sequence was $0.5 \mathrm{~mm}$, with a slice thickness of $1.0 \mathrm{~mm}$ and a slice gap of $0.5 \mathrm{~mm}$.

A summary of quantitative results for stenosis grading of these data is given in Table 3. In the same table, stenosis grades assessed by a radiologist from 3D TOF MIPs and 2D DSA projections are also included for comparison. Our model based method correlates better with DSA, which is the gold standard in many radiological studies, than the manual assessment from 3D TOF. Note that since we have not assumed a circular vessel shape, other shape characteristics such as the minimum/maximum diameters and cross-sectional area of the vessel can be computed, yielding additional quantitative information.

Although the performance has to be evaluated on a larger set, the algorithm performs well for non-severe stenoses or in absence of large flow artifacts. In Figure 4(b) we included an example of a severe stenosis. In this case, poststenotic flow artifacts and poor luminal signal showed that the almost fully automated method does not yield accurate results if the original data are of 

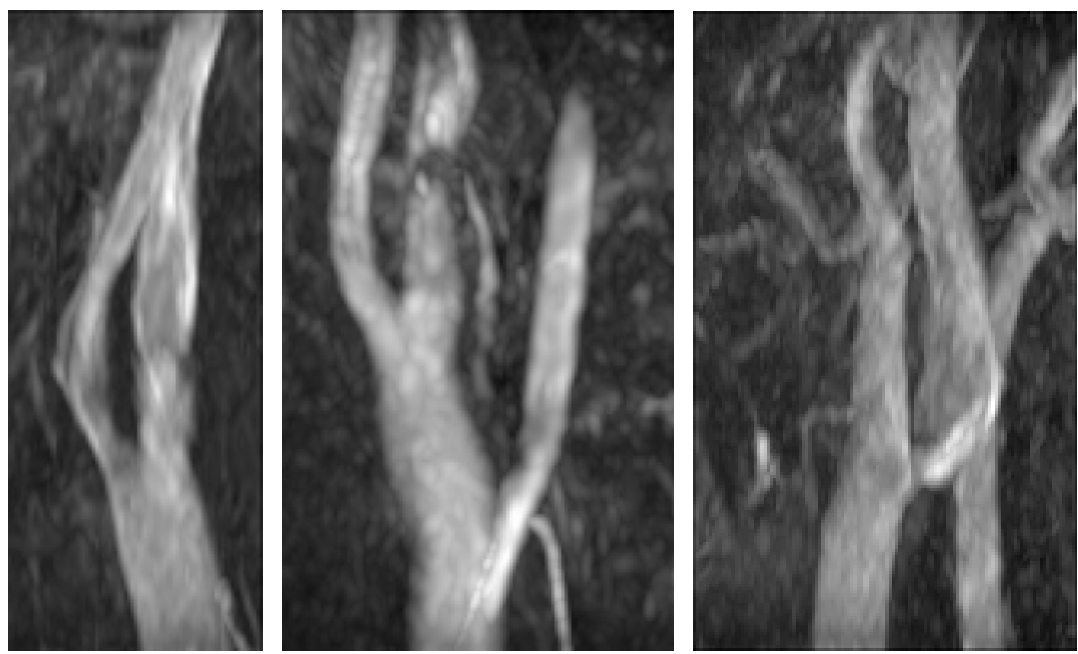

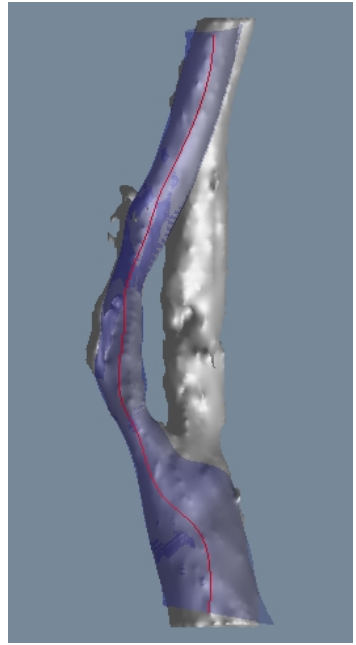

(a)

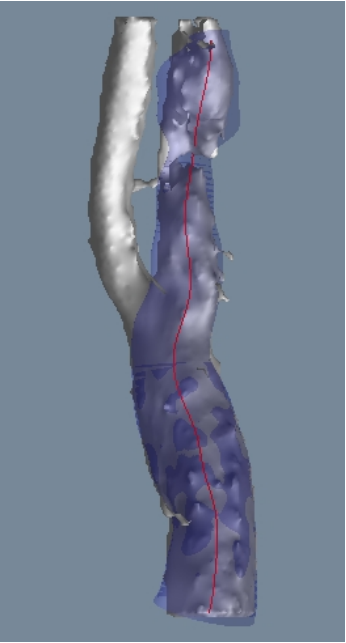

(b)

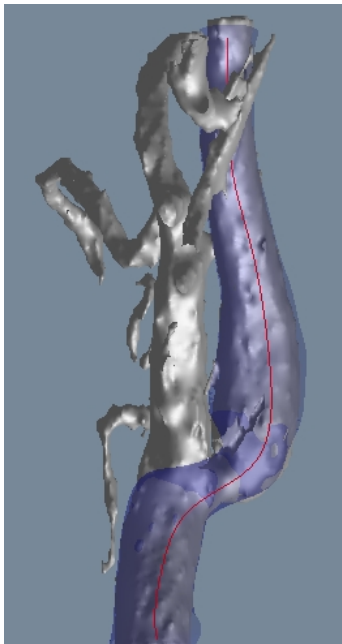

(c)

Fig. 4. Illustration on clinical data. The top row shows maximum intensity projections of 3D TOF datasets of the internal carotid artery (ICA). a) and b) correspond to the right and left ICA of the same patient and $c$ ) corresponds to the left ICA of a second patient. In the lower row we show the corresponding vessel models. The models are quite accurate for the two left ICA cases shown. For the right ICA, however, the presence of flow artifacts at the place of the severe stenosis (90\% graded on MRA and $53 \%$ on DSA according to NASCET) precluded following the vessel axis in the stenosis. 
poor quality (note that in this case the measurements of the operators in the 3D TOF and 2D DSA also show a large difference).

\section{Discussion}

We devised a method to perform quantitative diameter assessment at sub-voxel precision. The method shares some features with multiscale vessel enhancement algorithms based on eigenvalue analysis of the Hessian originally proposed by Koller et al. [16] and further modified by Sato et al. [17] and Lorenz et al. [18]. However, in these approaches a segmentation of the vasculature is obtained by estimating the vessel width as a function of the scale of maximum response. The accuracy of these algorithms is therefore a priori limited by the discretization of the scale parameter. This limits the applicability of these methods to give a general overview of the vasculature; for stenosis grading a more accurate approach is required. Aylward et al. [19] have suggested a method for vessel segmentation based on tracking the intensity ridges. The local vessel width is estimated using a line search technique to compute the scale of maximum response of a medialness function. Although this method can overcome the scale discretization problem, it still assumes that the vessel has a circular cross-section.

We have applied an algorithm which estimates the boundaries of the vessels using a flexible B-spline model which can be initialized using a small number of mouse clicks in a 3D surface display, and incorporates knowledge of the MR

\begin{tabular}{|c|c|c|c|c|c|}
\hline Criterion & Code & $\begin{array}{c}W O_{1} \\
\% D \pm C I_{99 \%}\end{array}$ & $\begin{array}{c}W O_{2} \\
\% D \pm C I_{99 \%}\end{array}$ & $\begin{array}{c}B O_{12} \\
\% D \pm C I_{99 \%}\end{array}$ & $\begin{array}{c}\text { MB } \\
\% D \pm C I_{99 \%}\end{array}$ \\
\hline \multirow{2}{*}{$\mathrm{CC}(78.5 \% \pm 0.65 \%)$} & 3D-TOF-1.0 & $69.8 \pm 3.47$ & $62.8 \pm 5.30$ & $66.3 \pm 3.20$ & $76.7 \pm 0.20$ \\
\hline & 3D-CEA-1.0 & $68.4 \pm 1.40$ & $62.8 \pm 4.10$ & $65.6 \pm 2.30$ & $76.0 \pm 0.22$ \\
\hline \multirow{2}{*}{ NASCET $(69.2 \% \pm 0.95 \%)$} & 3D-TOF-1.0 & $55.2 \pm 4.10$ & $39.1 \pm 7.52$ & $47.2 \pm 5.21$ & $67.6 \pm 0.14$ \\
\hline & $3 \mathrm{D}-\mathrm{CEA}-1.0$ & $55.8 \pm 3.30$ & $48.3 \pm 5.25$ & $52.1 \pm 3.38$ & $65.9 \pm 0.19$ \\
\hline
\end{tabular}

Table 1. Stenosis Grading Summary: carotid bifurcation phantom with asymmetric stenosis. Within $\left(W O_{1}, W O_{2}\right)$ and between observer $\left(\mathrm{BO}_{12}\right)$ variability of two experts compared to the model-based approach $(M B)$. The stenosis is given according to two stenosis indexes: the common carotid artery (CC) and NASCET indexes. The true values are indicated in the first column.

\begin{tabular}{lccc}
\hline Patient & \multicolumn{2}{c}{ 3D TOF 2D DSA 3D TOF (MB) } \\
& $\% D$ & $\% D$ & $\% D$ \\
\hline Fig. 4(a) & $28 \%$ & $36 \%$ & $38 \%$ \\
Fig. 4(b) & $90 \%$ & $53 \%$ & NA \\
Fig. 4(c) & $53 \%$ & $34 \%$ & $28 \%$ \\
\hline
\end{tabular}

Table 2. Stenosis grading of the carotids. Comparison of manual (3D TOF and DSA) and model-based (3D TOF). The stenosis index follows the NASCET criterion. 
image acquisition. Whereas the algorithm contains a scale selection procedure in the determination of the central vessel axis, this does not influence the diameter assessment. Also, the method can model a wide class of cross-sectional shapes. Although often it is assumed that vessels have a circular cross-section, ex vivo measurements [20] have shown that this assumption is rather simplistic and, especially at the stenosis, a wide variety of geometric shapes can be observed. Three dimensional approaches, as the one presented in this work, provide a basis for both the description of the actual cross-sectional shape and its quantification. They also allow definition of stenosis indexes based on cross-sectional area reduction which are more robust than those based on diameter reduction.

The method has been illustrated on phantom and patient data. In the phantom data the method obtained diameter and stenosis measurements with an accuracy which was significantly better than the experts. Results on the TOF MRA data of patients are promising. However, this imaging technique is prone to flow artifacts. A larger evaluation study, including state-of-the-art acquisition techniques like CE or blood pool agent (BPA) MRA which are less susceptible to flow artifacts, is planned to assess in which cases the proposed procedure can be used for reliable stenosis grading. The results presented in this study indicate that the proposed method has higher reproducibility and is more accurate than the laborious manual procedure of stenosis grading.

\section{References}

1. North American Symptomatic Carotid Endarterectomy Trial (NASCET) Steering Committe, "North American symptomatic carotid endarterectomy trial," Stroke, vol. 22, pp. 711-720, 1991. 358, 363

2. European Carotid Surgery Trialists' Collaborative Group, "Randomised trial of endarterectomy for recently symptomatic carotid stenosis: final results of the MRC European Carotid Surgery (ECST)," The Lancet, vol. 351, pp. 1379-87, 1998. 358

3. H. Cline, C. Dumoulin, W. Lorensen, S. Souza, and W. Adams, "Volume rendering and connectivity algorithms for MR angiography," MRM, vol. 18, pp. 384-394, 1991. 358

4. C. M. Anderson, J. S. Saloner, D. Tsuruda, L. G. Shapeero, and R. E. Lee, "Artifacts in maximum-intensity-projection display of MR angiograms," Am. J. Roent., vol. 154 , pp. 623-629, 1990. 358

5. S. Schreiner, C. B. Paschal, and R. L. Galloway, "Comparison of projection algorithms used for the construction of maximum intensity projection images," JCAT, vol. 20, no. 1, pp. 56-67, Jan-Feb 1996. 358

6. G. Farin, Curves and Surfaces for Computer Aided Geometric Design: A Practical Guide, Academic Press, 1990. 359

7. M. Kass, A. Witkin, and D. Terzopoulos, "Snakes: Active contour models," IJCV, vol. 1, no. 4, pp. 321-331, 1987. 359

8. T. McInerney and D. Terzopoulos, "Deformable models in medical image analysis: a survey," Med. Im. Anal., vol. 1, no. 2, pp. 91-108, 1996.359

9. W. Wesselink, Variational Modeling of Curves and Surfaces, Ph.D. thesis, Utrecht University, Computer Science Department, 1996. 359, 361 
10. A. F. Frangi, W. J. Niessen, K. L. Vincken, and M. A. Viergever, "Multiscale vessel enhancement filtering," in MICCAI98, A. Colchester W. M. Wells and S. Delp, Eds., 1998, vol. 1496 of LNCS, pp. 130-137. 359, 360

11. T. Lindeberg, "Feature detection with automatic scale selection," IJCV, vol. 30, no. 2, pp. 79-116, Nov. 1998. 360

12. L. Piegl and W. Tiller, The NURBS Book, Monographs in Visual Communication. Springer, 1996. 360, 362

13. R. M. Hoogeveen, C. J. G. Bakker, and M. A. Viergever, "Limits to the accuracy of vessel diameter measurement in MR angiography," J. Magn. Reson. Imag., vol. 8, pp. $1228-35,1998.361$

14. W. E. Lorensen and H. E. Cline, "Marching cubes: a high resolution 3D surface reconstruction algorith," Proc. SIGGRAPH'87, vol. 21, pp. 163-169, 1987. 362

15. R. F. Smith, B. K. Rutt, A. J. Fox, R. N. Rankin, and D. W. Holdsworth, "Geometric characterization of stenoted human carotid arteries," Acad. Radiol., vol. 3, pp. 898-911, 1996. 362, 363, 364

16. Th. Koller, G. Gerig, G. Székely, and D. Dettwiler, "Multiscale detection of Curvilinear Structures in 2-D and 3-D Image Data," in Proc. ICCV95, 1995, pp. 864-869, IEEE Computer Society Press. 366

17. Y. Sato, S. Nakajima, N. Shiraga, H. Atsumi, S. Yoshida, T. Koller, G. Gerig, and R. Kikinis, "Three-dimensional multi-scale line filter for segmentation and visualization of curvilinear structures in medical images," Med. Im. Anal., vol. 2, no. 2, pp. 143-168, June 1998. 366

18. C. Lorenz, I.-C. Carlsen, Buzug T.M., C. Fassnacht, and J. Weese, "Multi-scale line segmentation with automatic estimation of width, contrast and tangential direction in 2D and 3D medical images," in Proc. CVRMed-MRCAS'97, J. Troccaz, E. Grimson, and R. Mösges, Eds., 1997, LNCS, pp. 233-242, Springer-Verlag. 366

19. S. Aylward, E. Bullit, S. Pizer, and D. Eberly, "Intensity ridge and widths for tubular object segmentation and description," in Proc. IEEE/SIAM Workshop on Math. Meth. in Biomed. Im. Anal., 1996, pp. 131-8. 366

20. X. M. Pan, D. Saloner, L. M. Reilly, J. C. Bowersox, S. P. Murray, C. M Anderson, G. A. Gooding, and J. H. Rapp, "Assessment of carotid artery stenosis by ultrasonography, conventional angiography, and magnetic resonance angiography: correlation with ex vivo measurements of plaque stenosis," J. Vasc. Surg., vol. 21, pp. 82-89, 1995. 367

21. O. E. H. Elgersma, P. C. Buijs, A.F.J. Wüst, Y. van der Graaf, B. C. Eikelboom, and W. P. Th. M. Mali, "Assessment of maximum internal carotid artery stenosis: Rotational vs. conventional intra arterial digital substraction angiography," Radiol., accepted, 1999. 